\title{
Características dos Sistemas de Controle Gerencial das Pequenas e Médias Empresas do setor farmacêutico do Estado do Rio de Janeiro: Estudo de Casos
}

\author{
Antonio José Patrocínio Pereira \\ Mestrado em Ciências Contábeis pela Universidade do Estado do Rio de Janeiro - \\ UERJ \\ Centro Universitário Augusto Motta - UNISIUAM \\ E-mail: antoniojosepp@yahoo.com.br
}

\section{RESUMO}

O presente estudo tem por objetivo identificar a relação existente entre o controle gerencial abordado na literatura e o adotado por quatro pequenas e médias empresas do setor farmacêutico sediadas no estado do Rio de Janeiro. Devido às características e propósitos da pesquisa, adotou-se o método de estudos de casos, por ser aquele que possibilita alcançar maior nível de profundidade sobre o assunto e permite um melhor poder de análise de uma ou mais organizações, através da comparação destas com tipos ideais encontrados na literatura. Os resultados obtidos sugerem que os sistemas de controle gerencial utilizados nas empresas estudadas não atendem, em diversos aspectos, os fundamentos teóricos que nortearam este estudo. Por outro lado, observou-se que mesmo naquelas empresas onde foram encontrados sistemas de controle gerencial que, em alguns aspectos, apresentavam pontos relacionados com os vistos como relevantes na literatura estudada, quando examinados do ponto de vista do sistema como um todo, demonstravam desconhecimento acerca da importância de integração dos mecanismos de controle no alcance dos objetivos da organização.

Palavras-chave: Controle gerencial. Controle de gestão. Sistema de controle gerencial. PME.

Characteristics of Managerial Control Systems of Small and Medium Sized Companies of the Pharmaceutical Sector in Federal State of Rio de Janeiro: a Case Study

\begin{abstract}
The current study aims at identifying the relationship between the managerial control described in the specialized literature and the managerial control actually adopted by four small and medium-sized companies of the pharmaceutical sector located in the Federal State of Rio de Janeiro. Due to the characteristics and goals of this research, we have adopted the case study method, because this method is best suited for deeper analysis of one or more organizations, through the comparison of those with ideal types found in the literature. The obtained results suggest that the managerial control systems
\end{abstract}


used by the studied companies do not meet, in many aspects, the theoretical demands used as guidelines for the present study. On the other hand, we have observed that even in those companies where managerial control systems were found which, in some aspects, corresponded to the topics considered relevant in the studied literature, when they were examined from the point of view of the system as a whole, they showed a lack of knowledge concerning the importance of integration of the control mechanisms in reaching the objectives of the organization.

Key Words: Managerial Control. Management Control. Managerial Control System. Small and Medium Sized Companies.

\section{INTRODUÇÃO}

O tema controle de gestão experimentou uma considerável expansão conceitual a partir da década de 1950. Ao longo de todo esse período e, principalmente, durante a década de 1970, pode-se perceber o surgimento de novas abordagens que, valendo-se de uma perspectiva mais ampla do conceito de controle, passaram a incorporar conhecimentos oriundos de outras áreas (como, por exemplo: psicologia, sociologia e antropologia), com implicações marcantes no desenho e operacionalização dos sistemas de controle.

Em sintonia com esta temática, desenvolveu-se uma pesquisa com o objetivo de estudar a prática de planejamento e controle desenvolvida por empresas do setor farmacêutico, confrontando-a com a literatura disponível, levando-se em conta a característica e peculiaridade da atividade desenvolvida pelas empresas. Nessa linha, o estudo também tem a finalidade de responder ao seguinte questionamento:

- Qual a relação existente entre o controle gerencial adotado por pequenas e médias empresas farmacêuticas sediadas no estado do Rio de Janeiro e o que prescreve a literatura a respeito?

\section{CONTROLE GERENCIAL}

O termo "controle gerencial" deve muito de sua atual conotação ao estudo de Anthony (1965), no qual controle gerencial distingue-se de planejamento estratégico e controle operacional, estando situado em uma posição intermediária. O planejamento 
estratégico relaciona-se com a definição de metas e objetivos que englobam toda a organização no longo prazo. Em contrapartida, o controle operacional relaciona-se com as atividades que asseguram que as tarefas imediatas sejam realizadas, cabendo ao controle gerencial fazer a ligação entre esses dois níveis. Tal divisão apresentou duas vantagens: Colocou de lado a questão da determinação dos objetivos das organizações e os processos utilizados para a formulação e redefinição de estratégias e evitou a variedade de situações específicas que precisariam ser consideradas quando controles operacionais específicos são observados. Isto permitiu que o controle gerencial pudesse ser estudado sem deter-se em complicações desnecessárias. A definição clássica de Anthony (1965, p.17) "de que o controle gerencial é o processo pelo qual os administradores se asseguram que os recursos são obtidos e usados eficaz e eficientemente de acordo com os objetivos da organização", foi sendo aperfeiçoada ao longo dos anos, passando a incorporar elementos comportamentais. Mais recentemente, Anthony e Govindarajan (2002, p.34) definem controle gerencial como "o processo pelo qual os executivos influenciam os outros membros da organização para que obedeçam às estratégias adotadas". Este processo envolve diversas atividades tais como: a) planejar o que a organização deve fazer; b) coordenar as atividades das diversas partes da organização; c) comunicar as informações; d) avaliar informações; e) decidir se deve ser tomada uma decisão, ou que decisão tomar; e f) influenciar as pessoas para que alterem seu comportamento. Para os autores, o controle gerencial é um dos vários tipos das atividades de planejamento e controle que ocorre na organização. Na figura 1 podem ser visualizadas as relações gerais entre as funções de planejamento e controle. 


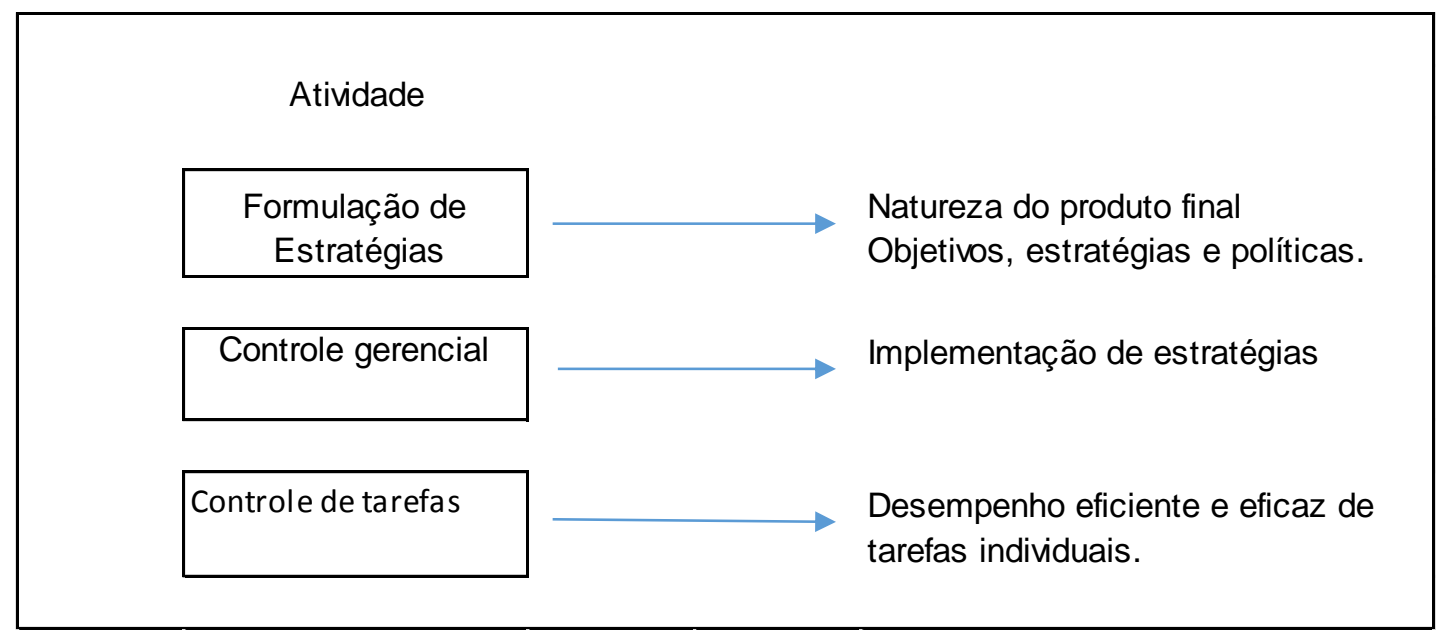

Figura 1 - Relações gerais entre as funções de planejamento e controle Fonte: Anthony e Govindarajan (2002, p. 34).

Para Anthony e Govindarajan (2002) a formulação de estratégias é o processo pelo qual se decide a adoção de novas estratégias e o controle gerencial é o processo pelo qual se decide a implementação de estratégias e a obediências a elas. Do ponto de vista de idealização de sistemas, a diferença mais importante entre a formulação de estratégias e o controle gerencial é que aquela (formulação de estratégias) é essencialmente assistemática. Quando surge um risco ou quando surge uma nova ideia, entra em cena a formulação de estratégias. Como os riscos (atuações diferentes de concorrentes no mercado, alterações de gostos de clientes) e oportunidades (inovações tecnológicas, novas tendências de comportamento de clientes e novas aplicações para produtos já existentes) não são detectados sistematicamente, isto é, em intervalos regulares, as decisões estratégicas são assistemáticas, podendo ocorrer a qualquer tempo.

A diferença mais importante entre o controle de tarefas e o controle gerencial está no fato de que muitos sistemas de controle de tarefas são científicos, e o controle gerencial não pode ser reduzido a uma ciência. Por definição, o controle gerencial compreende o comportamento dos executivos e comportamentos não podem ser expressos por meio de equações. Abrange tipos amplos de atividades, e os executivos decidem o que deve ser feito dentro dos limites das estratégias. O controle de tarefas abrange tarefas específicas para as quais não há julgamentos a serem feitos. 
Características dos Sistemas de Controle Gerencial das Pequenas e Médias Empresas do setor farmacêutico do Estado do Rio de Janeiro: Estudo de Casos Antonio José Patrocínio Pereira

Para Anthony e Govindarajan (2002) o propósito central de um sistema de controle gerencial (no qual o processo orçamentário é o elemento central) é assegurar, na medida do possível, a congruência dos objetivos. Para eles, a congruência dos objetivos de um processo significa que os atos e as atitudes que este leva as pessoas a adotar, de conformidade com seus próprios interesses, sejam também do interesse da organização.

Os autores destacam que mesmo sendo sistemático, o processo de controle não é mecânico na medida em que depende da interação entre as pessoas. Mencionam que os gerentes (e os funcionários, de modo geral) possuem objetivos pessoais, e o problema central do controle é induzi-los a agirem de tal maneira que ao procurarem alcançar seus objetivos pessoais, ajudem a alcançar os objetivos da organização. Isto é denominado de goal congruence, que significa que, tanto quanto possível, os objetivos dos membros da organização devem ser consistentes com os da organização como um todo. Alertam, contudo, que é impossível obter uma congruência perfeita. A impossibilidade de obter a perfeita congruência entre os objetivos individuais e os da organização decorreria da existência de processos informais que concorreriam com os sistemas formais, influenciando o comportamento humano dentro da organização.

Estes processos informais têm origem externa e interna à organização. Os fatores de origem externa se referem às normas de comportamento desejável que existem na sociedade da qual a organização faz parte, os quais podem ser específicos de uma determinada localidade, região ou país, ou então específicos de um determinado ramo de negócios. Dentre os fatores de origem interna, o mais importante é a cultura organizacional, ou seja, o conjunto de crenças, atitudes, normas, relações e valores que são explícitas ou implicitamente aceitos dentro da organização.

\subsection{Sistema de controle no contexto social e organizacional}

Gomes e Salas (2001) apresentam um modelo que procura integrar as ideias discutidas até agora e que consideram o controle dentro de uma perspectiva estratégica e organizacional, enfatizando três elementos dentro do processo de controle: o próprio sistema de controle, o contexto organizacional e o contexto social. 
Em primeiro lugar, percebe-se que o sistema de controle, propriamente dito, compreende: a estrutura e o processo de controle. A estrutura de controle está desenhada de acordo com as responsabilidades de cada gestor e compreende três elementos: sistema de medidas, sistema de informação e sistema de incentivos.

O processo de controle, por sua vez, compreende outros três elementos: a formulação de objetivos, o orçamento e a avaliação do desempenho. Dentro do sistema de controle encontram-se a contabilidade financeira e a contabilidade de gestão (sistemas de custos, planejamento e controle orçamentário). Além desses elementos, também são encontrados os sistemas de controle menos formalizados, onde se destacam: cultura organizacional, crenças, emoções, estilo de liderança, motivação, ética, ecologia, etc.

Em segundo lugar, o contexto organizacional em que o sistema de controle opera, influencia fortemente o desenho e o funcionamento do sistema. Este contexto organizacional compreende a estrutura, que facilita uma maior ou menor coordenação e eficiência das diversas unidades; a estratégia, as pessoas que fazem parte da organização e as relações interpessoais existentes entre elas, que favorecem maior ou menor motivação; bem como a cultura organizacional, que favorece uma maior ou menor identificação dos componentes da organização com esta.

Em terceiro lugar, o meio-ambiente social em que se desenvolve uma organização é, também, um fator fundamental para a compreensão do controle de gestão na prática. O contexto social inclui o mercado, o setor (clientes, fornecedores, concorrência, etc), o país (valores, instituições, história, nível de desenvolvimento) e a sociedade de determinado país, em seu todo (meio-ambiente tecnológico, cultural, político, demográfico, ecológico, etc). O meio ambiente é, igualmente, um importante mecanismo de controle, mas a diferença dos fatores anteriores é, geralmente, muito mais complexa e, assim, difícil de ser controlada.

A figura 2 permite visualizar o que foi exposto. 
Características dos Sistemas de Controle Gerencial das Pequenas e Médias Empresas do setor farmacêutico do Estado do Rio de Janeiro: Estudo de Casos

Antonio José Patrocínio Pereira

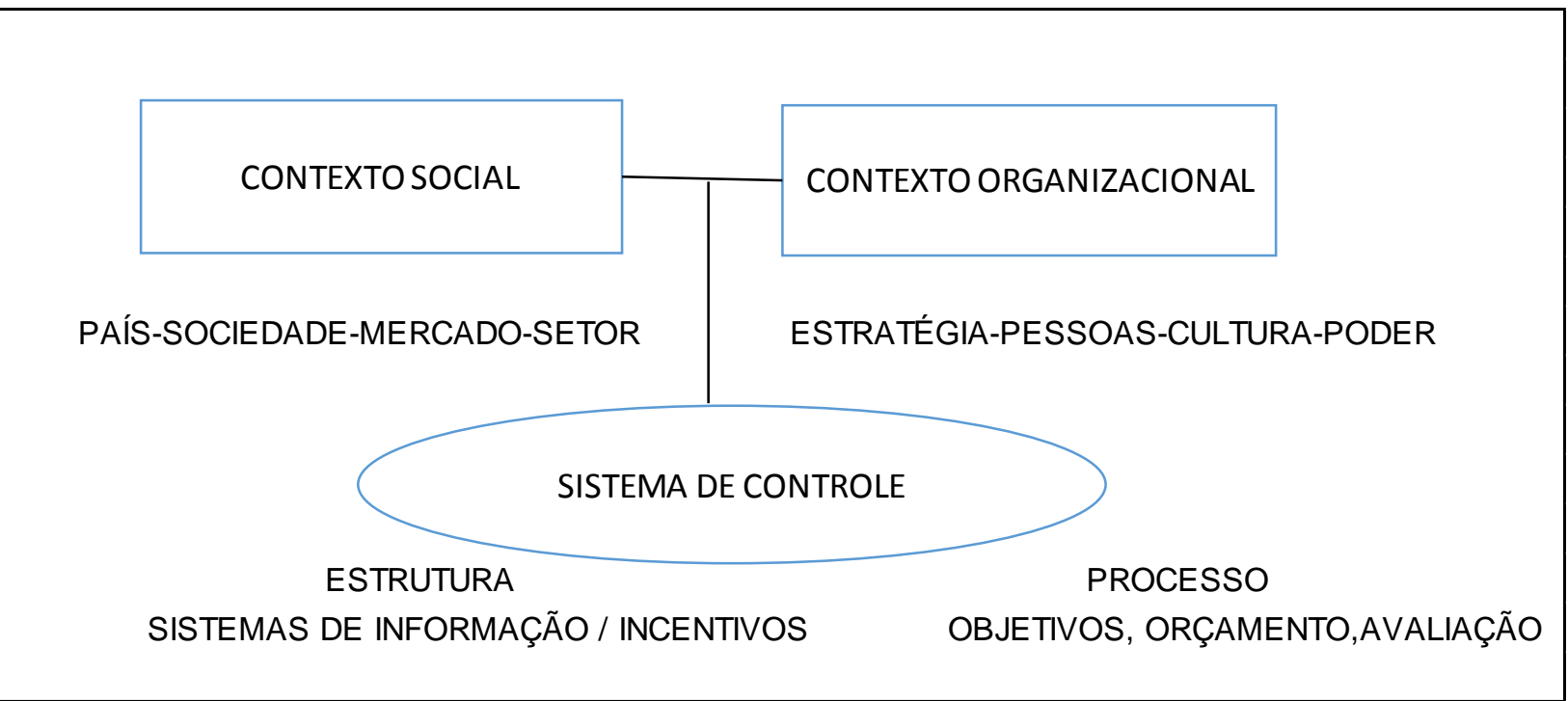

Figura 2 - Vinculação do sistema de controle com o contexto social e organizacional.

Fonte: Amat e Gomes (1995).

\subsection{Tipos de controle organizacional}

Os sistemas de controle das organizações sofreram significativas mudanças na última década, consequência das transformações que vêm ocorrendo, constantemente, no contexto sócio-econômico mundial e mesmo em seus ambientes internos. Gomes e Salas (2001) fundamentam as mudanças ocorridas nos sistemas de controle, afirmando que: (a) as mudanças no contexto social e, em particular, a crise dos mecanismos de controle social, durante este período, associados à crise econômica mundial e seu impacto na redução da rentabilidade; (b) o desenvolvimento do neoliberalismo e as bruscas alterações verificadas no cenário internacional, que deram origem à introdução de sistemas de controle essencialmente financeiros, os quais passaram a ser institucionalizados e legitimados como sistemas de controle por excelência.

Escolher este ou aquele sistema de controle decorre principalmente das características, da dimensão e do contexto social onde está inserida a organização. Assim, o tamanho da organização e a formalização são características fundamentais para explicar o tipo de sistema de controle de uma organização, ou seja, quanto maior esta for, maior terá de ser a formalização do sistema de controle. A análise do contexto social onde a empresa está inserida é fundamental para se determinar o grau de dinamismo e a 
concorrência. Gomes e Salas (2001, p.56) citam que "à medida que o contexto social é mais dinâmico e competitivo, tende-se a uma maior descentralização e a utilização de sistemas de controle financeiros orientados à gestão de resultado".

Partindo dessas considerações e, em função da combinação de mecanismos utilizados pelas organizações, Gomes e Salas in Pereira, 2006 apresentam, conforme quadro 1, diferentes tipos de controle organizacional: controle familiar, controle burocrático, controle por resultados e controle ad-hoc.

\begin{tabular}{|c|c|c|c|}
\hline Dimensão & Contexto Local & Contexto Internacional & Formalização \\
\hline PEQUENO PORTE & CONTROLE FAMILIAR & CONTROLE AD-HOC & $\begin{array}{c}\text { LIMITADA } \\
\text { FORMALIZAÇÃO }\end{array}$ \\
\hline GRANDE PORTE & $\begin{array}{c}\text { CONTROLE } \\
\text { BUROCRÁTICO }\end{array}$ & $\begin{array}{c}\text { CONTROLE POR } \\
\text { RESULTADOS }\end{array}$ & $\begin{array}{c}\text { ELEVADA } \\
\text { FORMALIZAÇÃO }\end{array}$ \\
\hline & CENTRALIZADA & DESCENTRALIZADA & \\
\hline
\end{tabular}

Quadro 1 - Tipologia de controle organizacional.

Fonte: Gomes e Salas (2001, p.56).

O controle familiar normalmente é encontrado em organizações de pequeno porte e que atuam em um contexto social local, tendo um tipo de controle mais informal. A deficiência deste tipo de controle evidencia-se quando a empresa se volta para um cenário mais competitivo.

O controle ad-hoc pode ser observado também em organizações de menor porte, porém voltadas ao contexto internacional e baseia-se, fundamentalmente, na utilização de mecanismos não formais que promovam o autocontrole. Podem ser encontrados em organizações cuja cultura está baseada no individualismo e na busca de consenso, que normalmente atuam em um ambiente marcado pelo dinamismo e pela complexidade.

No que diz respeito ao controle burocrático, ele pode ser observado em organizações de grande porte que apresentam uma cultura avessa às mudanças, centralizadoras do poder e fechadas para o exterior, mas voltadas para um contexto local. As limitações deste tipo de controle vêm à tona quando a organização ingressa em um mercado mais competitivo e hostil. 
Características dos Sistemas de Controle Gerencial das Pequenas e Médias Empresas do setor farmacêutico do Estado do Rio de Janeiro: Estudo de Casos

Antonio José Patrocínio Pereira

Os controles por resultados abrangem as organizações que atuam em um contexto social internacional e os controles são geralmente baseados em sistemas de controle financeiro e podem ser observados, principalmente, em organizações que atuam em contextos sociais muito competitivos, que possuem cultura organizacional voltada aos resultados.

\subsection{Perspectivas de controle}

Para Amat e Gomes (1995) dentro do conceito de controle distinguem-se duas perspectivas claramente diferenciadas: em primeiro lugar, uma perspectiva limitada do que significa o controle na organização, baseado normalmente em aspectos financeiros; e em segundo lugar, uma perspectiva mais ampla do controle onde se considera também o contexto em que ocorrem as atividades e, em particular, os aspectos ligados a estratégia, estrutura organizacional, comportamento individual, cultura organizacional e meio-ambiente (Quadro 1).

\begin{tabular}{|c|c|c|}
\hline Aspecto & Perspectiva Limitada & Perspectiva Ampla \\
\hline Filosofia & $\begin{array}{c}\text { Controle de cima para baixo } \\
\text { Controle como sistema }\end{array}$ & $\begin{array}{c}\text { Controle realizado por todos } \\
\text { Controle como atitude }\end{array}$ \\
\hline Énfase & Cumprimento & Motivação, auto controle \\
\hline Conceito de controle & $\begin{array}{c}\text { Medição dos resultados baseado } \\
\text { análise de desvios e geração de } \\
\text { relatórios. Desenho, técnicas de } \\
\text { gestão. }\end{array}$ & $\begin{array}{c}\text { Desenvolvimento de uma } \\
\text { consciência estratégica } \\
\text { orientada para o } \\
\text { aperfeiçoamento contínuo. } \\
\text { Melhora da posição competitiva }\end{array}$ \\
\hline $\begin{array}{c}\text { Consideração do contexto social, } \\
\text { organizacional e humano. }\end{array}$ & $\begin{array}{c}\text { Limitado. Ênfase no desenho de } \\
\text { aspectos técnicos. Normas } \\
\text { rígidas, padrões valores } \\
\text { monetários. }\end{array}$ & $\begin{array}{c}\text { Amplo, Contexto sociala, } \\
\text { sociedade, cultura, emoções, } \\
\text { valores. }\end{array}$ \\
\hline Fundamento teórico & Economia, Engenharia & $\begin{array}{c}\text { Antropologia, Sociologia, } \\
\text { Psicologia }\end{array}$ \\
\hline Mecanismo de controle & $\begin{array}{c}\text { Controle baseado no resultado } \\
\text { da contabilidade de Gestão. }\end{array}$ & $\begin{array}{c}\text { Formal e informal. Outras } \\
\text { variáveis, aprendizado. }\end{array}$ \\
\hline
\end{tabular}

Quadro 2 - Perspectivas de Controle

Fonte: Adaptado de Amat (1995, p.27)

A partir de uma perspectiva mais ampla, pode-se considerar que o controle organizacional é exercido através de diferentes mecanismos que tratam de motivar, 
Características dos Sistemas de Controle Gerencial das Pequenas e Médias Empresas do setor farmacêutico do Estado do Rio de Janeiro: Estudo de Casos

Antonio José Patrocínio Pereira

orientar e influir para que o comportamento individual e organizacional seja o mais conveniente para alcançar os objetivos a longo prazo dos membros da organização.

\subsection{Vinculação do sistema de controle com a estrutura}

Quanto maior for a descentralização, mais difícil e custoso se torna o controle. Isto pode explicar a preferência pela centralização e implicar em um desafio para a delegação de tarefas aos níveis inferiores para definir a autoridade formal e indicar o processo operacional desejado e os meios para adquirir recursos.

Um sistema de controle em uma empresa descentralizada deve ser mais sofisticado e formalizado, com predomínio de sistemas de controle financeiros e orientados à atuação de cada responsável. No quadro a seguir, apresenta-se um resumo das principais diferenças no desenho do sistema de controle, segundo o grau de descentralização.

\begin{tabular}{|c|c|c|}
\hline Tópicos & Estrutura Centralizada & Estrutura Descentralizada \\
\hline Sistemas de controle & $\begin{array}{l}\text { Pouco sofisticado. Mecanismos } \\
\text { informais. Normas rígidas }\end{array}$ & $\begin{array}{l}\text { Muito sofisticado. Mecanismos } \\
\text { formais. Controle financeiro }\end{array}$ \\
\hline Indicadores de controle & $\begin{array}{l}\text { Qualitativos. Nem sempre } \\
\text { orientados a resultados }\end{array}$ & $\begin{array}{l}\text { Quantitativos. Orientados a } \\
\text { resultados }\end{array}$ \\
\hline Sistema de informação & $\begin{array}{l}\text { Orientado ao resultado global. } \\
\text { Pouco orientado ao controle de } \\
\text { responsabilidades. }\end{array}$ & $\begin{array}{l}\text { Orientado a tomada de decisões } \\
\text { e ao controle da atuação dos } \\
\text { responsáveis }\end{array}$ \\
\hline Processo de planejamento & $\begin{array}{l}\text { Inexistente ou intuitivo. Quando } \\
\text { ocorre pode ter caráter } \\
\text { burocrático }\end{array}$ & $\begin{array}{c}\text { Fundamental para lograr } \\
\text { consenso sobre os objetivos } \\
\text { globais. }\end{array}$ \\
\hline Sistema de avaliação & $\begin{array}{l}\text { Baseado em informação não } \\
\text { financeira. }\end{array}$ & $\begin{array}{c}\text { Baseado em informação } \\
\text { financeira e não financeira. }\end{array}$ \\
\hline
\end{tabular}

Quadro 3 - Desenho de um sistema de controle em função do grau de descentralização Fonte: Amat (1993, p. 82)

Segundo Gomes e Salas (2001), o tipo de estrutura organizacional influi igualmente no sistema de controle em função da forma adotada: funcional, divisional ou matricial. Em uma estrutura funcional deve prevalecer o controle burocrático e orientado para a eficiência. O sistema de controle de gestão deve dar ênfase ao registro da informação, limitando a utilização como mecanismo de controle e ficando reduzido à alocação de recursos a diversos centros de responsabilidade. 
Características dos Sistemas de Controle Gerencial das Pequenas e Médias Empresas do setor farmacêutico do Estado do Rio de Janeiro: Estudo de Casos

Antonio José Patrocínio Pereira

Já na estrutura divisionalizada a preferência deve recair no controle por resultados, com ênfase nos indicadores relacionados à eficácia, dada sua maior descentralização e orientação para o mercado. O controle deve fornecer informação para a tomada de decisões, planejamento e avaliação de desempenho dos responsáveis pelos centros de responsabilidades.

E, por fim, numa estrutura matricial deve-se encontrar uma combinação de controle por resultados e controle ad hoc, devido à complexidade das atividades de cada centro. Diante da forte inter-relação torna-se difícil avaliar, isoladamente, a ação de cada responsável sem considerar a influência de outros centros.

Neste caso, a contabilidade de gestão deve ser um facilitador no processo de negociação dos objetivos e do orçamento a ser utilizada de forma bastante flexível na avaliação do desempenho dos responsáveis pelos centros. O quadro 3 resume bem estes pontos.

\begin{tabular}{|c|c|c|c|}
\hline Tópicos & Funcional & Divisional & Matricial \\
\hline $\begin{array}{c}\text { Sistema de } \\
\text { controle }\end{array}$ & Burocrático & Resultados & $\begin{array}{c}\text { Ad hoce } \\
\text { resultados }\end{array}$ \\
\hline $\begin{array}{c}\text { Indicadores de } \\
\text { controle }\end{array}$ & $\begin{array}{c}\text { Quantitativos. } \\
\text { Eficiência }\end{array}$ & $\begin{array}{c}\text { Quantitativos. } \\
\text { Eficácia }\end{array}$ & $\begin{array}{c}\text { Qualitativos } \\
\text { quantitativos }\end{array}$ \\
\hline $\begin{array}{c}\text { Sistemas de } \\
\text { informação }\end{array}$ & $\begin{array}{c}\text { Mensuração de } \\
\text { gastos }\end{array}$ & $\begin{array}{c}\text { Resultado do } \\
\text { centro }\end{array}$ & Não financeiro \\
\hline $\begin{array}{c}\text { Processo de } \\
\text { Planejamento }\end{array}$ & $\begin{array}{c}\text { Alocação de } \\
\text { recursos }\end{array}$ & $\begin{array}{c}\text { Fundamental para } \\
\text { avaliação }\end{array}$ & $\begin{array}{c}\text { Assegurar } \\
\text { coordenação }\end{array}$ \\
\hline $\begin{array}{c}\text { Sistema de } \\
\text { avaliação }\end{array}$ & $\begin{array}{c}\text { Importância } \\
\text { limitada }\end{array}$ & $\begin{array}{c}\text { Resultado dos } \\
\text { centros }\end{array}$ & Resultado global \\
\hline
\end{tabular}

Quadro 4 - Sistema de controle em função da estrutura Fonte: Gomes e Salas (2001, p.130).

\subsection{Sistema de controle em função da estratégia}

Ainda, de acordo com Gomes e Salas (2001), um sistema de controle deve-se adequar às características do contexto social traçadas para a organização e, como consequência, à estratégia da organização. Através da vinculação do orçamento anual com a estratégia de longo prazo, assegura-se que os diferentes centros atuem, separadamente, para alcançar seus objetivos setoriais e consequentemente os objetivos e as diretrizes globais. 
Características dos Sistemas de Controle Gerencial das Pequenas e Médias Empresas do setor farmacêutico do Estado do Rio de Janeiro: Estudo de Casos

Antonio José Patrocínio Pereira

Para os autores, os sistemas devem facilitar a informação para formular e avaliar a priori uma estratégia, adequando a sua implantação. Na realidade as empresas outorgam excessiva ênfase à informação financeira, mas não utilizam toda a potencialidade da informação contábil na formulação da estratégia e no controle estratégico, dando preferência à informação qualitativa, tanto interna como externa, devido a algumas deficiências apresentadas para serem utilizadas no processo estratégico de diferentes ordens.

\section{METODOLOGIA DA PESQUISA}

Para Gomes (2006), um caso é uma descrição de uma situação real do mundo dos negócios, experimentado por uma determinada organização ou pessoa, em certo momento, envolvendo um problema, uma decisão, um desafio ou uma oportunidade a ser considerada por alguém. $O$ autor menciona que o caso configura uma importante ferramenta no entendimento da teoria.

Permite conectar a teoria com a prática e oportuniza o desenvolvimento de avanços teóricos.

Para Zikmund (1997), a vantagem primária de um estudo de caso é permitir que uma organização ou entidade seja investigada em profundidade, com atenção para detalhes.

\subsection{Variáveis da pesquisa}

O objetivo primário das variáveis é possibilitar a sistematização dos dados, "reduzindo problemas comuns encontrados em trabalhos que utilizam o método de estudo de casos" (Gomes, 1983, p.70).

No estudo realizado foram consideradas as seguintes variáveis dos componentes do sistema de controle gerencial: (a) Objetivos organizacionais; (b) Processo Orçamentário; (c) Sistema de contabilidade gerencial; (d) Medidas de avaliação de desempenho; (e) Evolução dos sistemas de controle gerencial. Os componentes do sistema de controle gerencial foram operacionalizados como: 
Características dos Sistemas de Controle Gerencial das Pequenas e Médias Empresas do setor farmacêutico do Estado do Rio de Janeiro: Estudo de Casos

Antonio José Patrocínio Pereira

a) Objetivos organizacionais: a verificação da estratégia adotada pela empresa para o atendimento dos objetivos da organização dar-se-á através de:

1. Avaliação do processo de definição e comunicação dos objetivos à organização;

2. Avaliação da dinâmica do processo de mudança e revisão dos objetivos com base na percepção dos entrevistados;

3. Avaliação do tipo de integração entre os objetivos e as metas;

4. Verificação do processo usado pela empresa na avaliação do atendimento dos objetivos organizacionais em confronto com os resultados apresentados.

A análise deste primeiro componente (objetivos organizacionais) de um sistema de controle gerencial, objetiva classificar as empresas em termos de proximidade ou afastamento em relação ao sistema de controle gerencial eficaz referenciado na literatura. Anthony e Govindarajan (2002) destacam que na formulação de uma estratégia empresarial existe uma nítida distinção entre objetivos e metas. Lorange e Morton (1974) asseveram que a preocupação principal é com a ligação entre objetivos e metas. Hofstede (1978) ressalta que se deve procurar corrigir o processo e não os resultados, o que implica em revisão periódica dos objetivos. A turbulência ambiental força a existência de novos objetivos, implicando em ter que se avaliar sistematicamente o atendimento dos objetivos organizacionais, segundo Hofstede (1981). Flamholtz (1979) menciona que a coordenação de diversas partes da organização envolve comunicação, de modo que todas compreendam o que se espera delas.

Para isto o sistema necessita ser formalizado de maneira a facilitar a integração dos objetivos com as metas.

b) Processo orçamentário: a análise deste componente do sistema de controle gerencial objetiva avaliar a adequação das metas organizacionais ao sistema de planejamento e controle da empresa através de:

1. Verificação da importância atribuída pela empresa à participação das pessoas no processo orçamentário; 
Características dos Sistemas de Controle Gerencial das Pequenas e Médias Empresas do setor farmacêutico do Estado do Rio de Janeiro: Estudo de Casos

Antonio José Patrocínio Pereira

2. Análise dos componentes básicos do orçamento (propósito, conteúdo, horizonte temporal, processo de revisão formal, acompanhamento e fontes de dados), quanto a sua adequação ao processo orçamentário da empresa;

3. Avaliação do processo orçamentário quanto a sua flexibilidade e capacidade de adaptação a fatores externos (evolução, aperfeiçoamento, processo de revisão);

4. Verificação da existência de programas de desenvolvimento de pessoal ligados ao processo orçamentário (programas de educação contínua em técnicas orçamentárias). A análise do processo orçamentário objetivando classificar as empresas estudadas em termos de proximidade ou afastamento em relação ao sistema de controle gerencial referenciado como eficaz na literatura, baseia-se nos fundamentos teóricos citados a seguir.

c) Sistema de contabilidade gerencial: a verificação deste componente do sistema de controle gerencial objetiva avaliar o estado e a natureza do sistema, bem como a estrutura de responsabilidade através da:

1. Avaliação do conteúdo, importância e tipos de relatórios gerados pela contabilidade gerencial;

2. Avaliação da velocidade de resposta (feedback) aos relatórios gerenciais;

3. Percepção dos gerentes quanto à adequação dos relatórios gerenciais às necessidades de informação da empresa;

4. Percepção dos gerentes quanto a dificuldades na implantação de relatórios gerenciais na empresa;

5. Análise da definição e compreensão do conceito de centro de responsabilidade utilizado pela empresa;

6. Avaliação dos mecanismos de preços de transferência e sua revisão entre os centros de responsabilidades.

7. Avaliação do processo de acompanhamento do centro de responsabilidade;

8. Avaliação do método de custeio utilizado.

d) Medida de avaliação de desempenho: a análise deste componente do sistema de controle gerencial tem por objetivo avaliar a existência e validade de instrumentos formais de desempenho das unidades organizacionais, que são utilizados pela empresa 
para reforçarem comportamentos positivos e corrigir desvios no alcance dos objetivos da organização. Isto será efetuado através da:

1. Verificação da abrangência dos propósitos e das medidas formais, usados no processo de avaliação de desempenho projetados pela empresa;

2. Verificação da existência e validade dos padrões e procedimentos formais utilizados no processo de avaliação de desempenho;

3. Verificação da relevância do sistema formal de avaliação e recompensa utilizado no processo de controle gerencial (promoções e incentivos financeiros derivados do desempenho observado).

A análise deste componente de controle gerencial tem como objetivo a classificação das empresas estudadas em termos de proximidade ou afastamento em relação ao sistema de controle gerencial referenciado como eficaz na literatura, levando em consideração que a instabilidade do meio-ambiente faz com que as medidas baseadas em unidades monetárias se tornem insuficientes como variáveis de controle (Lorange e Morton, 1974). Muitas atividades apresentam resultados que só podem ser medidos em termos qualitativos; se não existirem medidas indiretas para mensurar os resultados, o controle das atividades torna-se uma questão de avaliação subjetiva (Hofstede, 1981). Os padrões, planos e objetivos definidos durante o processo orçamentário tornam-se pontos de referência para o sistema de controle (Maciariello e Kirby, 1994). O propósito de um sistema de controle eficaz é prover feedback como uma base para o processo de avaliação de desempenho (função informacional da medida). Além disso, o simples ato de medir pode influenciar o comportamento das pessoas na organização, pela tendência de se concentrar mais atenção nos fatores sujeitos à mensuração em detrimento daqueles não sujeitos (função processo) (Flamholtz, 1979).

e) Evolução dos sistemas de controle gerencial: a análise deste componente tem por objetivo avaliar a evolução do sistema de controle gerencial da empresa, verificando a adaptabilidade dos diversos mecanismos de controle ao meio ambiente externo.

Também tem por objetivo obter informações sobre as técnicas de gestão empregadas pela empresa. Isto será feito através de: 
Características dos Sistemas de Controle Gerencial das Pequenas e Médias Empresas do setor farmacêutico do Estado do Rio de Janeiro: Estudo de Casos

Antonio José Patrocínio Pereira

1. Avaliação do sistema de controle gerencial quanto a sua flexibilidade e capacidade de adaptação a fatores externos (evolução, aperfeiçoamento, processo de revisão).

2. Verificação dos instrumentos de controle gerencial utilizados pelas empresas. A classificação das empresas em termos de proximidade ou afastamento em relação aos pontos vistos na literatura sobre controle gerencial, leva em conta a necessidade dos sistemas serem modificados para refletirem a evolução de novos padrões organizacionais e da turbulência ambiental (Lorange e Morton, 1974).

\subsection{Critério de seleção das empresas estudadas}

Dadas limitações de ordem financeira, foram escolhidas quatro pequenas e médias empresas do setor farmacêutico localizadas no estado do Rio de Janeiro.

\subsection{Método de coleta de dados}

Tendo em vista o grau de profundidade da análise que se pretendeu empreender e o número limitado de empresas a serem estudadas, optou-se pela aplicação de questionários através de entrevista pessoal conduzida pelo próprio pesquisador.

O questionário elaborado foi organizado da seguinte forma: uma primeira parte, composta na sua maioria de perguntas abertas, refere-se aos sistemas de controle gerencial projetados pelas empresas e o processo por elas desenvolvido com vistas à descrição do sistema implantado. Entrevistou-se a pessoa indicada ou a que faz parte da direção como sendo a responsável direta pelo sistema de controle gerencial em uso.

Além disso, foram coletados, também, dados secundários, quer no âmbito destas empresas por ocasião da realização das entrevistas, quer através de fontes externas como jornais, revistas, internet, etc., de modo a estudar as diferenças existentes entre as características organizacionais e os sistemas de controle projetados por estas entidades, bem como avaliar algumas questões fornecidas pelos entrevistados. 
Características dos Sistemas de Controle Gerencial das Pequenas e Médias Empresas do setor farmacêutico do Estado do Rio de Janeiro: Estudo de Casos Antonio José Patrocínio Pereira

\section{DESCRIÇÃO DOS CASOS}

\subsection{EMEFARMA RIO}

A EMEFARMA RIO é uma distribuidora de produtos farmacêuticos voltada para o varejo, com sólida experiência no mercado do Estado do Rio de Janeiro. Em 29 de agosto de 2006 foi realizada uma entrevista com o Sr. Jony Anderson Tavares de Souza, responsável pela área administrativa da empresa, em conjunto com seu irmão Sergio Tavares de Souza, responsável pela área de marketing e vendas. Ambos são diretores executivos da empresa e fazem parte da $2^{\underline{a}}$ geração no processo sucessório da mesma.

De acordo com os entrevistados, a missão da empresa é oferecer produtos que promovam saúde e bem-estar, e soluções em logística integrada, buscando resultados eficazes para valorização da vida. A empresa possui nos seus quadros 93 funcionários, tanto na área administrativa como da área de marketing, e seu faturamento anual previsto para 2006 é de 32 milhões de reais. Em agosto de 1988 começa a história da empresa, no bairro de Monte

Castelo, no município de Nova Iguaçu, Rio de Janeiro. Seu idealizador e fundador, Eurico F. de Souza, instala-se numa pequena casa e inicia a distribuição de medicamentos para o município de Nova Iguaçu.

Atualmente, devido ao seu constante crescimento, a EMEFARMA RIO está situada na Rodovia Presidente Dutra, em Nova Iguaçu, numa área total de $6000 \mathrm{~m} 2$, onde foram realizados significativos investimentos em infra-estrutura, logística, tecnologia de última geração e capacitação profissional.

A EMEFARMA RIO distribui medicamentos de marca, similares, genéricos, etc., cosméticos, perfumaria, alimentos secos e health care. Com uma equipe de venda periodicamente atualizada, realizam-se visitas regulares aos clientes, apresentando o que há de mais moderno no segmento farmacêutico, campanhas e condições comerciais competitivas. 
Características dos Sistemas de Controle Gerencial das Pequenas e Médias Empresas do setor farmacêutico do Estado do Rio de Janeiro: Estudo de Casos

Antonio José Patrocínio Pereira

\subsection{A Nossa Drogaria}

A Nossa Drogaria é uma empresa de gestão familiar. Hoje está sob o comando dos filhos e netos de Jayme Pereira de Souza, seu fundador. Atua no mercado do Rio de Janeiro há 45 anos e possui 10 lojas distribuídas na baixada fluminense e zonas norte e oeste do município do Rio de Janeiro.

Em 14 de setembro de 2006 foi realizada uma entrevista com o Sr. Eduardo Pereira de Souza, diretor responsável pelas áreas administrativa e financeira da empresa, que com seu primo Leandro Pereira, diretor responsável da área de Marketing e Vendas, são os executivos responsáveis pela empresa e pertencem a $3^{\underline{a}}$ geração no processo sucessório. Todavia, seus pais são seus conselheiros e ficam mais voltados para a estratégia da empresa, principalmente, atuando mais num contexto social (país, sociedade, mercado e setor).

De acordo com o entrevistado a empresa possui, atualmente, 499 funcionários e seu faturamento não foi informado devido à posição estratégica adotada pela empresa. A Nossa Drogaria foi fundada em 1960, com o nome de Drogaria Nova, por Jayme Pereira de Souza e mais dois sócios.

Em 1979 a empresa deu um grande passo, inaugurando sua maior loja, a Matriz, localizada na Rua José de Alvarenga, Duque de Caxias - RJ. A partir de então todas as lojas passaram a pertencer somente a Jayme e seus filhos, com o nome de A Nossa Drogaria. Em 1991, Jayme Pereira, com 73 anos faleceu, deixando-a com seus filhos e netos.

Em 1996 foi inaugurada a primeira loja da rede em Shopping Center, em Nova Iguaçu - TOP Shopping. Entre 1997 e 1998 a empresa inaugurou três lojas - Vilar dos Teles, Santa Cruz e Penha. Atualmente está com dez lojas.

\subsection{Grupo Marlécio}

Em 18 de setembro de 2006 realizou-se uma entrevista com o Sr. Jéferson Nascimento Lima, assessor do proprietário da empresa Sr. Marlécio, objeto do estudo de caso e diretor da rede Max Center (Drogarias Max e Padrão). 
De acordo com o entrevistado, o grupo Marlécio possui atualmente 40 lojas sob a forma de sociedade comercial por cotas de responsabilidade limitada, utilizando-se da marca MAXCENTER. Conta em seus quadros com 1200 funcionários em todo o Estado do Rio de Janeiro, e o montante do faturamento não foi informado por questão de estratégia adotada na empresa.

No início da década de 1980, quatro empresários: Sr Luiz Carlos; Sr.Veras; Sr. João Vargas e o Sr. Marlécio, do ramo de farmácia e drogaria, constataram a necessidade de realizarem propaganda em rádio, busdoor e, posteriormente, em televisão e mídia em geral, de suas farmácias e drogarias. O custo era alto, surgiu então a ideia de usarem o mesmo nome de fantasia, nas suas lojas, isto é, DROGARIAS MAX.

Por volta de 1997, quando o trabalho já estava bastante divulgado, verificou-se a necessidade de criar uma nova marca, pois surgiam naquele momento outras empresas de franquia no ramo de farmácias e drogarias, consolidando o sistema no mercado. Então, foi criada a marca PADRÃO, hoje com 80 lojas. A mencionada marca já nasceu em berço esplêndido, pois a MAXCENTER, que conta atualmente com 160 franqueados, já havia adquirido todo o know-how no desenvolvimento do sistema de franquia, no ramo de farmácias e drogarias, logo, seu crescimento foi rápido.

\subsection{N.A. da Silva}

A N.A. da Silva é uma empresa de gestão familiar, atualmente, sob o comando do Sr. Nilson Antonio da Silva. Atuando no mercado do varejo farmacêutico, desde a década de 1960, conta com 06 lojas distribuídas estrategicamente nos municípios de Itaguaí e Seropédica, também denominada como a costa verde do estado.

Em 30 de setembro de 2006 foi realizada uma entrevista com o proprietário e fundador Sr. Nilson Antonio da Silva, diretor responsável pela gestão das empresas, que está preparando seu filho Sr. Nilson Antonio da Silva Junior para sucedê-lo na empresa.

De acordo com o entrevistado, a empresa conta atualmente com 400 funcionários e seu planejamento de vendas para 2006 é de $R \$ 10$ milhões. 
Características dos Sistemas de Controle Gerencial das Pequenas e Médias Empresas do setor farmacêutico do Estado do Rio de Janeiro: Estudo de Casos

Antonio José Patrocínio Pereira

Conforme o entrevistado, há 3 anos a N.A da Silva filiou-se a Rede Atual com um número aproximado de 70 lojas licenciadas, partindo do entendimento de que somente uma marca forte pode agregar valor de natureza comercial e comportamental, exigindo de seus participantes atitudes coerentes com os objetivos comuns no alcance das mudanças e oportunidades. Empresários, profissionais especialistas, consultorias, distribuidoras, laboratórios interagem no projeto e constatam que a visão se alarga, bem como consolida estratégias vitoriosas que conquistam, a cada passo, o apoio e a atenção que todo projeto sério tende a obter.

\begin{tabular}{|c|c|c|c|c|}
\hline Características & Emefarma & A Nossa Drogaria & Grupo Marlécio & N.A da Siva \\
\hline $1-$ Rede & Independente & Própria & MAX & ATUAL \\
\hline $\begin{array}{c}2-\text { Setor de } \\
\text { atividade }\end{array}$ & Distribuidora & Varejo & Varejo & Varejo \\
\hline $\begin{array}{c}3-\text { Sede } \\
4-\text { No de } \\
\text { estabelecimentos }\end{array}$ & Nova Iguaçu & Duque de Caxias & Rio de Janeiro & Itaguaí \\
\hline $\begin{array}{c}5-\text { Data de } \\
\text { fundação }\end{array}$ & 1 & 10 & 40 & 7 lojas \\
\hline $\begin{array}{c}6-\text { Faturamento } \\
\text { previsto anual }\end{array}$ & 32 milhões R\$ $(19$ a) & $1960(45$ a) & $1980(26$ a) & $1960(45$ a) \\
\hline $\begin{array}{c}7-\text { Número } \\
\text { funcionários }\end{array}$ & 93 & - & 1200 & 10 milhões R\$ \\
\hline $\begin{array}{c}8-\text { Porte da } \\
\text { Empresa }\end{array}$ & Médio Porte & Médio Porte & Médio Porte & Pequeno Porte \\
\hline $\begin{array}{c}9-\text { Diversificação } \\
\text { negócios }\end{array}$ & Não diversificada & Não diversificada & Não diversificada & Não diversificada \\
\hline $\begin{array}{c}10-\text { Processo } \\
\text { Sucessório }\end{array}$ & $2^{\text {a geração }}$ & $3^{\text {a geração }}$ & Fundador & Fundador \\
\hline
\end{tabular}

Figura 3 - Resumo das características organizacionais das empresas estudadas

\section{ANÁLISE DOS RESULTADOS DOS COMPONENTES DO SISTEMA DE CONTROLE}

No quadro, a seguir, apresenta-se um sumário dos resultados das variáveis utilizadas na análise dos componentes do sistema de controle gerencial pelas empresas estudadas: 
Características dos Sistemas de Controle Gerencial das Pequenas e Médias Empresas do setor farmacêutico do Estado do Rio de Janeiro: Estudo de Casos

Antonio José Patrocínio Pereira

a) Objetivos organizacionais: as empresas não desenvolvem uma estratégia empresarial que se aproxime dos modelos eficazes mencionados na literatura;

b) Processo orçamentário: A Nossa Drogaria desenvolveu processos que se aproximam da literatura, entretanto, a EMEFARMA, GRUPO MARLÉCIO, N.A. DA SILVA, encontram-se afastadas do que preceitua a literatura e a participação das pessoas no processo é restrita.

c) Sistema de Contabilidade Gerencial: A EMEFARMA e A NOSSA DROGARIA apresentam velocidade adequada nos relatórios gerados pela administração de forma satisfatória, mas se encontram afastadas quanto às demais.

d) Medidas de Avaliação de desempenho e evolução dos sistemas de controle: Os sistemas de controle gerencial dessas empresas guardam certa distância dos procedimentos administrativos compatíveis com o indicado na literatura.

\begin{tabular}{|c|l|}
\hline \multicolumn{2}{|c|}{ ANÁLISE DOS COMPONENTES SISTEMA DE CONTROLE } \\
\hline \multicolumn{2}{|c|}{ VARIÁVEIS DOS COMPONENTES DO SISTEMA DE CONTROLE GERENCIAL. } \\
\hline $\begin{array}{c}\text { Objetivos } \\
\text { Organizacionais }\end{array}$ & $\begin{array}{l}\text { a) Somente uma empresa desenvolve planejamento estratégico direcionado para } \\
\text { crescimento, preocupação com a definição, comunicação e revisão dos objetivos, } \\
\text { integração dos objetivos com metas, avaliação dos resultados alcançados em } \\
\text { confronto com os objetivos determinados. } \\
\text { b) Nas demais, os objetivos são voltados para o curto prazo sendo o orçamento } \\
\text { financeiro considerado o instrumento principal. }\end{array}$ \\
\hline $\begin{array}{c}\text { Processo } \\
\text { Orçamentário }\end{array}$ & $\begin{array}{l}\text { a)Não consideram os efeitos motivacionais do orçamento no comportamento das } \\
\text { pessoas. Todavia, numa empresa destaca-se certa participação dos gerentes, na } \\
\text { elaboração do orçamento. } \\
\text { b) Nas demais, embora, mais restrita, percebe-se maior participação da pessoas em } \\
\text { apresentar metas quantitativas. }\end{array}$ \\
\hline $\begin{array}{c}\text { Sistema } \\
\text { Gontabilidade } \\
\text { Gerencial }\end{array}$ & $\begin{array}{l}\text { a)Duas empresas estão implantando ou modernizando os sistemas de contabilidade } \\
\text { gerencial, voltados para planejamento e controle. } \\
\text { b) Uma empresa possui processos formais de acompanhamento do desempenho } \\
\text { dos seus centros de responsabilidade. }\end{array}$ \\
\hline $\begin{array}{c}\text { Medidas de } \\
\text { Avaliação e } \\
\text { Desempenho. }\end{array}$ & $\begin{array}{l}\text { a) Limitam-se às variações orçamentárias e os mecanismos de controle são } \\
\text { Medidas de Avaliação incapazes de identificar os objetivos ou comportamentos } \\
\text { relevantes. }\end{array}$ \\
\hline
\end{tabular}

Quadro 5 - Análise dos componentes do sistema de controle 
Características dos Sistemas de Controle Gerencial das Pequenas e Médias Empresas do setor farmacêutico do Estado do Rio de Janeiro: Estudo de Casos

Antonio José Patrocínio Pereira

\section{CONSIDERAÇÕES FINAIS}

Conforme definido na introdução, procurou-se responder a questão formulada por meio dos estudos de casos realizados nas quatro empresas pequenas e médias do setor farmacêutico pesquisadas, concluindo-se:

As quatro empresas podem ser enquadradas dentro de uma tipologia organizacional familiar e o controle é mais informal.

Pode-se considerar que nas empresas o controle se encontra sob uma perspectiva limitada, baseado em aspectos financeiros.

Todas as empresas apresentam sistema de controle centralizado.

Todas as empresas possuem sistema de controle funcional, em termos de estrutura.

O sistema de controle utilizado nas empresas, em função da estratégia, apresenta-se mais voltado para o controle operacional.

As quatro empresas estudadas ainda não apresentam todas as características consideradas como eficazes na literatura.

As empresas estudadas ainda não utilizam o controle gerencial como processo para que os executivos influenciem outros membros da organização a obedecerem as estratégias adotadas.

\section{REFERÊNCIAS}

AMAT, J.M. e GOMES, J.S. 1995. Controle de gestão: uma perspectiva global. Congresso Internacional de Custos, IV. Campinas, 1995.

ANTHONY, R.N. 1965. Planing and control systems: a framework for analysis. Boston, Harvard Business School, $180 \mathrm{p}$.

Atlas, $\overline{1019 p}$.

; GOVINDARAJAN, V. 2002. Sistemas de controle gerencial. São Paulo,

BNDES-Banco Nacional de desenvolvimento Econômico e Social. Critério oficial de classificação das MPME. Disponível em: http://www.bndes.gov.br, acesso em 26/11/2006. 
Características dos Sistemas de Controle Gerencial das Pequenas e Médias Empresas do setor farmacêutico do Estado do Rio de Janeiro: Estudo de Casos

FLAMHOLTZ, E.G. 1979. Organizational control systems as managerial tool. California Management Review, 22(2):71-84 p.

GOMES, J.S. 1983. Um estudo exploratório sobre controle gerencial em empresas estatais brasileiras. Rio de Janeiro, RJ. Tese de doutorado. COPPEAD/UFRJ, $358 \mathrm{p}$.

; SALAS, J.M.A. 2001. Controle de Gestão: uma abordagem contextual e organizacional. 3를. ed. São Paulo, Atlas, 192 p.

São Paulo, 97 p.

2006. O Método de Estudo de Caso aplicado à gestão de negócios.

HOFSTEDE, 1978. Geert. The poverty of management control philosophy. Academy of Management Review, 45-61 p. Accounting, Organizations and Society 6(3): 193-211.

LORANGE, P. e MORTON, M.S.S. 1974. A framework for management control systems. Sloan management review, 16(1):41-56.

MACIARIELLO, J.A e KIRBY, C.J. 1994. Management control systems: using adaptive systems to attain control. Upper Saddle River, Prentice Hall, 662 p.

PEREIRA, A.J.P. e GOMES, J.S. 2006. Sistema de Controle Gerencial em Empresas Brasileiras Internacionalizadas: Estudo de Caso Petroflex. Anais do XXX EnANPAD, Salvador, 2006, p. 268. 219 p.

ZIKMUND, W.G. 1997. Business research methods. Fort Worth, Dryden Press, Data de Submissão: 28/12/2006 Data de Aceite: 27/08/2007 